\title{
Maximum Power Point Characteristics of Generalized Heat Engines with Finite Time and Finite Heat Capacities
}

\author{
Abhishek Khanna ${ }^{1,2}$ and Ramandeep S. Johal ${ }^{1}$ \\ ${ }^{1}$ Department of Physical Sciences, Indian Institute of Science Education and Research Mohali, Sector 81, Manauli, Mohali, \\ Punjab 140306, India \\ ${ }^{2}$ Elite Course Theoretical and Mathematical Physics, Ludwig Maximillian University, D-80333 Munich, Germany
}

Correspondence should be addressed to Ramandeep S. Johal, rsjohal@iisermohali.ac.in

Received 19 September 2012; Revised 27 November 2012; Accepted 27 November 2012

Academic Editor: M. A. Rosen

Copyright ( 2012 A. Khanna and R. S. Johal. This is an open access article distributed under the Creative Commons Attribution License, which permits unrestricted use, distribution, and reproduction in any medium, provided the original work is properly cited.

\begin{abstract}
We revisit the problem of optimal power extraction in four-step cycles (two adiabatic and two heat-transfer branches) when the finite-rate heat transfer obeys a linear law and the heat reservoirs have finite heat capacities. The heat-transfer branch follows a polytropic process in which the heat capacity of the working fluid stays constant. For the case of ideal gas as working fluid and a given switching time, it is shown that maximum work is obtained at Curzon-Ahlborn efficiency. Our expressions clearly show the dependence on the relative magnitudes of heat capacities of the fluid and the reservoirs. Many previous formulae, including infinite reservoirs, infinite-time cycles, and Carnot-like and non-Carnot-like cycles, are recovered as special cases of our model.
\end{abstract}

\section{Introduction}

Curzon-Ahlborn efficiency, $\eta_{\mathrm{CA}}=1-\sqrt{T_{2} / T_{1}}$, where $T_{1}$ and $T_{2}$ are the reservoir temperatures [1], is regarded as a landmark result of finite-time thermodynamics. It models the effect of irreversibilities due to finite rate of heat transfer on the performance of heat engines. Such models are termed as endoreversible. The flow of heat between the working fluid and the reservoirs is assumed to be Newtonian. All the components maintain an internal equilibrium and losses due to friction, and heat leak are assumed to be negligible. Many authors [2-10] extended and clarified the scope of this model. In fact, various other models of heat engines also yield maximum power output at efficiencies very close to CA efficiency [11-15]. This universal-like behaviour of efficiency has been recently analysed using Bayesian probabilities in the case of quantum models of engines [16].

In this paper, we revisit the problem of optimal performance with "linear" irreversibilities of finite time and finite heat reservoirs in classical models of engines. This question was addressed in [7] using a Lagrangian formalism, for a one-component working fluid without assuming an equation of state. However, the generic problem with finite reservoirs could not be solved in a closed form. Then Gordon [8] used an ancillary device of intermediate reservoirs to arrive at a closed form solution. In particular, it was shown [8] that for Carnot-like engines, finiteness of reservoirs has no effect on the efficiency at maximum power, and it is still at Curzon-Ahlborn efficiency. The case of other non-Carnot heat engines (such as Otto cycle, Joule-Brayton cycle, and Atkinson cycle) was discussed by using an infinite chain of Carnot cycles.

The present analysis of the problem is based on the following features: (a) the working fluid is a classical ideal gas; (b) the total cycle time as well as switching time is given. We consider a generic four-step cycle with two adiabatic and two heat-transfer branches, which follow a polytropic process with a constant heat capacity $C>0$. The process is described by the relation $T V^{x}=k$. Here, $T$ is the temperature of the working fluid, $V$ is the volume of the working fluid, and $k$ is an arbitrary constant. Consequently, many popular heat engines like Carnot cycle $(x=0)$, Otto cycle $(x \rightarrow \infty)$, and Joule-Brayton $(x=-1)$ can be incorporated in this study.

Our key results may be listed as follows. For ideal gas as the working fluid and the generic four-step cycle (including 
Carnot and non-Carnot heat cycles), the power of the engine is maximum at CA efficiency, even for finite reservoirs. Many special cases like infinite time and infinite reservoirs [11], finite time and infinite reservoirs with isothermal branches [1] or polytropic branches can be described. We note that closed form expressions for work can be derived without invoking a device like an intermediate reservoir [8]. Further, the heat capacities of the working fluid and the (finite) reservoirs enter explicitly in the expressions, and their relative sizes appear as the respective ratios of heat capacities. Also, the explicit expressions of work for these special cases clearly show that the irreversibilities of finite time and/or finite reservoirs reduce the maximum amount of work extracted as compared with infinite time and infinite reservoirs cases.

\section{The Model}

2.1. Temperature Profiles. First of all, we calculate how the temperatures of the two bodies change, when kept in contact with one another for some time. The heat transfer between the bodies is Newtonian, and during heat transfer, their heat capacities remain constant.

Let the two bodies denoted as $A$ and $B$ have heat capacities, $C_{A}$ and $C_{B}$, respectively. The bodies are kept in thermal contact with one another from time, $t=0$ to $t=t_{1}$. The temperatures of the two bodies at any time $t$ are $T_{A}(t)$ and $T_{B}(t)$, respectively, and the coefficient of heat conductivity is $K$.

At time $t$, the heat flow between bodies $A$ and $B$ should be equal to heat gained (lost) by one or heat lost (gained) by the other. Therefore,

$$
K\left[T_{A}(t)-T_{B}(t)\right] d t=-C_{A} d T_{A}(t)=C_{B} d T_{B}(t) .
$$

Solving the above equations, temperature profiles of the two bodies at time $t$ are calculated as

$$
\begin{aligned}
& T_{A}(t)=T_{A}(0)+\frac{\gamma_{1}}{\gamma}\left(1-e^{-\gamma t}\right), \\
& T_{B}(t)=T_{B}(0)+\frac{\gamma_{2}}{\gamma}\left(1-e^{-\gamma t}\right),
\end{aligned}
$$

where

$$
\begin{gathered}
\gamma=K\left(\frac{1}{C_{A}}+\frac{1}{C_{B}}\right), \\
\gamma_{1}=\frac{-K}{C_{A}}\left(T_{A}(0)-T_{B}(0)\right), \\
\gamma_{2}=\frac{K}{C_{B}}\left(T_{A}(0)-T_{B}(0)\right) .
\end{gathered}
$$

2.2. The Cycle. We have two finite heat reservoirs at initial temperatures $T_{1}(0)$ and $T_{2}(0)$ and heat capacities are $C_{1}$ and $C_{2}$, respectively. A working fluid is chosen to extract work from the reservoirs. The time period for one working cycle is $t_{2}$. One working cycle consists of the following steps.

(i) Working fluid is brought in contact with the hot reservoir from time $t=0$ to $t_{1}$. The fluid expands along a path with heat capacity $C$. Consequently, temperature of the hot reservoir reduces from $T_{1}(0)$ to $T_{1}\left(t_{1}\right)$, and that of the working fluid rises from $T(0)$ to $T^{-}\left(t_{1}\right)$.

(ii) The fluid is allowed to expand adiabatically. This step is assumed to be instantaneous and takes negligible time. As a result, the temperature of the working fluids jumps from $T^{-}\left(t_{1}\right)$ to $T^{+}\left(t_{1}\right)$.

(iii) The fluid is brought in contact with the cold reservoir from time $t_{1}$ to $t_{2}$. The fluid contracts along a path with heat capacity $C$. Consequently, temperature of the cold reservoir rises from $T_{2}\left(t_{1}\right)$ to $T_{2}\left(t_{2}\right)$ and that of the working fluid reduces from $T^{+}\left(t_{1}\right)$ to $T^{-}\left(t_{2}\right)$.

(iv) Working fluid is allowed to contract adiabatically. This step is also assumed to take negligible time. As a result, the temperature of the working fluids jumps from $T^{-}\left(t_{2}\right)$ to $T(0)$, completing the cycle.

Using (2)-(6), the temperature profiles of the reservoirs and the working fluid in different steps can be described as follows:

(i) $0<t<t_{1}$ :

$$
\begin{aligned}
& T_{1}(t)=T_{1}(0)+\frac{\lambda_{1}}{\lambda}\left(1-e^{-\lambda t}\right), \\
& T(t)=T(0)+\frac{\lambda_{2}}{\lambda}\left(1-e^{-\lambda t}\right),
\end{aligned}
$$

where

$$
\begin{aligned}
\lambda & =K_{1}\left(\frac{1}{C_{1}}+\frac{1}{C}\right), \\
\lambda_{1} & =\frac{-K_{1}}{C_{1}}\left[T_{1}(0)-T(0)\right], \\
\lambda_{2} & =\frac{K_{1}}{C}\left[T_{1}(0)-T(0)\right] .
\end{aligned}
$$

(ii) $t=t_{1}$ :

$$
\begin{gathered}
\lim _{t \rightarrow t_{1}^{-}} T(t)=T(0)+\frac{\lambda_{2}}{\lambda}\left(1-e^{-\lambda t}\right)=T^{-}\left(t_{1}\right), \\
\lim _{t \rightarrow t_{1}^{+}} T(t)=T^{+}\left(t_{1}\right), \quad T^{+}\left(t_{1}\right)<T^{-}\left(t_{1}\right) .
\end{gathered}
$$

(iii) $t_{1}<t<t_{2}$ :

$$
\begin{aligned}
& T(t)=T^{+}\left(t_{1}\right)+\frac{\beta_{1}}{\beta}\left(1-e^{-\beta\left(t-t_{1}\right)}\right), \\
& T_{2}(t)=T_{2}\left(t_{1}\right)+\frac{\beta_{2}}{\beta}\left(1-e^{-\beta\left(t-t_{1}\right)}\right) .
\end{aligned}
$$

Since $T_{2}\left(t_{1}\right)=T_{2}(0)$, this implies

$$
T_{2}(t)=T_{2}(0)+\frac{\beta_{1}}{\beta}\left(1-e^{-\beta\left(t-t_{1}\right)}\right),
$$




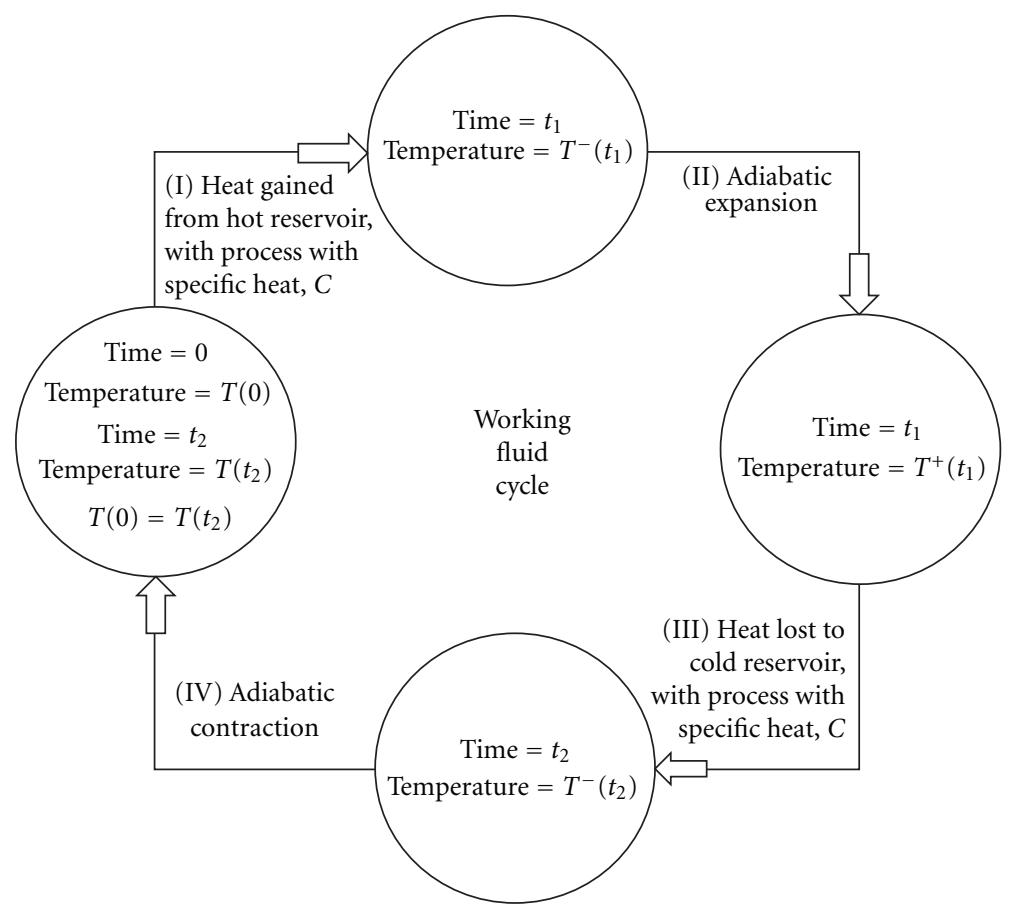

FIGURE 1: Heat cycle of the working fluid.

where

$$
\begin{aligned}
& \beta=K_{2}\left(\frac{1}{C_{2}}+\frac{1}{C}\right), \\
& \beta_{1}=\frac{-K_{2}}{C}\left(T^{+}\left(t_{1}\right)-T_{2}\left(t_{1}\right)\right), \\
& \beta_{2}=\frac{K_{2}}{C_{2}}\left(T^{+}\left(t_{1}\right)-T_{2}\left(t_{1}\right)\right) .
\end{aligned}
$$

(iv) $t=t_{2}$ :

$$
\begin{gathered}
\lim _{t \rightarrow t_{2}^{-}} T(t)=T^{+}\left(t_{1}\right)+\frac{\beta_{1}}{\beta}\left(1-e^{-\beta\left(t-t_{1}\right)}\right)=T^{-}\left(t_{2}\right), \\
\lim _{t \rightarrow t_{2}^{+}} T(t)=T(0) .
\end{gathered}
$$

The complete cycle is depicted in Figure 1.

We will now use the above temperatures to evaluate the work performed by the engine. Due to the cyclic process, the first law of thermodynamics implies that the net heat exchanged by the working fluid during the cycle equals the work performed. Thus

$$
W=\int_{T(0)}^{T^{-}\left(t_{1}\right)} C d T+\int_{T^{+}\left(t_{1}\right)}^{T^{-}\left(t_{2}\right)} C d T .
$$

Since specific heat is not a function of temperature,

$$
W=C\left[T^{-}\left(t_{1}\right)-T(0)\right]+C\left[T^{-}\left(t_{2}\right)-T^{+}\left(t_{1}\right)\right] .
$$

Using (9), (10), and (14), we obtain

$$
W=C \frac{\lambda_{2}}{\lambda}\left(1-e^{\lambda t_{1}}\right)+C \frac{\beta_{1}}{\beta}\left(1-e^{-\beta\left(t_{2}-t_{1}\right)}\right) .
$$

Substituting values for $\lambda, \lambda_{2}, \beta$ and $\beta_{1}$ from (8) and (13), we have

$$
\begin{aligned}
W= & \frac{C C_{1}}{C+C_{1}}\left[T_{1}(0)-T(0)\right]\left(1-e^{-K_{1}\left(\left(1 / C_{1}\right)+(1 / C)\right) t_{1}}\right) \\
& -\frac{C C_{2}}{C+C_{2}}\left[T^{+}\left(t_{1}\right)-T_{2}(0)\right]\left(1-e^{-K_{2}\left(\left(1 / C_{2}\right)+(1 / C)\right)\left(t_{2}-t_{1}\right)}\right) .
\end{aligned}
$$

Heat absorbed from the hot reservoir $\left(Q_{1}\right)$ is given by

$$
\begin{aligned}
Q_{1}=\int_{T(0)}^{T^{-}\left(t_{1}\right)} C d T= & \frac{C C_{1}}{C+C_{1}}\left[T_{1}(0)-T(0)\right] \\
& \times\left(1-e^{-K_{1}\left(\left(1 / C_{1}\right)+(1 / C)\right) t_{1}}\right) .
\end{aligned}
$$

There is no change in the entropy of the working fluid after one cycle. Since the change in the entropy of the working fluid occurs only in the nonadiabatic branches ((i) and (iii)),

$$
\oint d S=C \int_{T(0)}^{T^{-}\left(t_{1}\right)} \frac{d T}{T}+C \int_{T^{+}\left(t_{1}\right)}^{T^{-}\left(t_{2}\right)} \frac{d T}{T}=0,
$$

which yields

$$
T^{+}\left(t_{1}\right)=\frac{T^{-}\left(t_{1}\right) T^{-}\left(t_{2}\right)}{T(0)} .
$$

Here, it should be noticed that no heat leakage or entropy production is considered in this model. 


\section{Case I: Finite Time Studies with Infinite Reservoirs}

In this section, we will first optimize the work per cycle over the initial temperature $T(0)$ of the working fluid and then with respect to the switching time $t_{1}$.

Under the infinite reservoirs condition $\left(C_{1} \rightarrow \infty, C_{2} \rightarrow\right.$ $\infty)$, the temperatures of hot and the cold reservoirs remain fixed at values $T_{1}(0)$ and $T_{2}(0)$, respectively. Using (9), (14), and (22) and substituting $\lambda=K_{1} / C$ and $\beta=K_{2} / C$, we can calculate temperature of working fluid after the adiabatic step as

$$
\begin{aligned}
T^{+}\left(t_{1}\right)=[ & T_{2}(0)\left(1-e^{-\left(K_{2} / C\right)\left(t_{2}-t_{1}\right)}\right) \\
& \left.\times\left(T_{1}(0)+T(0) e^{-\left(K_{1} / C\right) t_{1}}-T_{1}(0) e^{-\left(K_{1} / C\right) t_{1}}\right)\right] \\
\times & {\left[T(0)-T_{1}(0) e^{-\left(K_{2} / C\right)\left(t_{2}-t_{1}\right)}\right.} \\
& \left.+\left(T_{1}(0)-T(0)\right) e^{-\left(K_{1} / C\right) t_{1}-\left(K_{2} / C\right)\left(t_{2}-t_{1}\right)}\right]^{-1}
\end{aligned}
$$

Substituting the above expression for $T^{+}\left(t_{1}\right)$ in (19), the work obtained is given by

$$
\begin{aligned}
W= & C\left(T_{1}(0)-T(0)\right)\left(1-e^{-\left(K_{1} / C\right) t_{1}}\right) \\
& \times\left[1-\frac{T_{2}(0)\left(1-e^{-\left(K_{2} / C\right)\left(t_{2}-t_{1}\right)}\right)}{\alpha}\right],
\end{aligned}
$$

where for convenience, we have defined

$$
\begin{aligned}
\alpha= & T(0)-T_{1}(0) e^{-\left(K_{2} / C\right)\left(t_{2}-t_{1}\right)} \\
& +\left(T_{1}(0)-T(0)\right) e^{-\left(K_{1} / C\right) t_{1}-\left(K_{2} / C\right)\left(t_{2}-t_{1}\right)} .
\end{aligned}
$$

On using (20) with the infinite reservoir condition, and (24), the efficiency of the heat engine $\left(\eta=W / Q_{1}\right)$ is given by

$$
\eta=1-\frac{T_{2}(0)\left(1-e^{-\left(K_{2} / C\right)\left(t_{2}-t_{1}\right)}\right)}{\alpha} .
$$

Now we will optimize the work per cycle with respect to initial temperature of the working medium, for a fixed switching time, and later, optimise with respect to the switching time also

$$
\left(\frac{\partial W}{\partial T(0)}\right)_{t_{1}}=0
$$

Solving the above equation, we observe that at maximum work,

$$
\begin{aligned}
\alpha= & \left(1-e^{-\left(K_{2} / C\right)\left(t_{2}-t_{1}\right)}\right) \sqrt{T_{1}(0) T_{2}(0)} . \\
T(0)= & {\left[\sqrt{T_{1}(0) T_{2}(0)}\left(1-e^{-\left(K_{2} / C\right)\left(t_{2}-t_{1}\right)}\right)\right.} \\
& \left.+T_{1}(0) e^{-\left(K_{2} / C\right)\left(t_{2}-t_{1}\right)}\left(1-e^{-\left(K_{1} / C\right) t_{1}}\right)\right] \\
& \times\left[1-e^{-\left(K_{1} / C\right) t_{1}-\left(K_{2} / C\right)\left(t_{2}-t_{1}\right)}\right]^{-1} .
\end{aligned}
$$




\section{Case II: Finite Time Studies with Finite Reservoirs}

In this section, we carry out the optimisation of work under the constraints of both finite reservoirs and finite cycle time. Solving similarly as in Section 3, the work becomes optimal when the initial temperature of the fluid is given by

$$
\begin{aligned}
T(0)= & {\left[\sqrt{T_{2}(0) T_{1}(0)}\left(C+C_{1}\right)\left(1-e^{-\beta\left(t_{2}-t_{1}\right)}\right)\right.} \\
& \left.+C_{1} T_{1}(0) e^{-\beta\left(t_{2}-t_{1}\right)}\left(1-e^{-\lambda t_{1}}\right)\right] \\
& \times\left[C\left(1-e^{-\lambda t_{1}}\right)+C_{1}\left(1-e^{-\lambda t_{1}-\beta\left(t_{2}-t_{1}\right)}\right)\right]^{-1} .
\end{aligned}
$$

The optimal work is explicitly given as

$$
\begin{aligned}
W_{\text {opt }}= & {\left[C_{1} C_{2}\left(\sqrt{T_{1}(0)}-\sqrt{T_{2}(0)}\right)^{2}\right.} \\
& \left.\times\left(1-e^{-\lambda t_{1}}\right)\left(1-e^{-\beta\left(t_{2}-t_{1}\right)}\right)\right] \\
& \times\left[C C_{1}\left(1-e^{-\lambda t_{1}}\right)+C C_{2}\left(1-e^{-\beta\left(t_{2}-t_{1}\right)}\right)\right. \\
& \left.\quad+C_{1} C_{2}\left(1-e^{-\lambda t_{1}-\beta\left(t_{2}-t_{1}\right)}\right)\right]^{-1} .
\end{aligned}
$$

The impact of finiteness of time can be seen on the work obtained, in Figure 2. It is shown that when time is large enough, the maximum work obtained is approximately equal to that in infinite time case (43). The efficiency at optimal work is again found to be CA value. To the best of our knowledge, only for Carnot-like cycles with isothermal branches $(C \rightarrow \infty)[8]$, it has been shown that finiteness of the reservoirs does not affect the efficiency at maximum power. Here, we have seen that the generic heat cycle with two adiabatic and two polytropic branches obtains maximum power at the CA efficiency. This efficiency is still unaffected by the switching time, even with the finite reservoirs.

We may further maximize the work with respect to the switching time:

$$
\frac{\partial W_{\mathrm{opt}}}{\partial t_{1}}=0
$$

The optimal switching time is the solution to the following equation:

$$
\lambda e^{-\lambda t_{1}}\left(1-e^{-\beta\left(t_{2}-t_{1}\right)}\right)^{2}=\beta e^{-\beta\left(t_{2}-t_{1}\right)}\left(1-e^{-\lambda t_{1}}\right)^{2},
$$

where $\lambda$ and $\beta$ are given by (8) and (13). The impact of finiteness of reservoirs on the optimal switching time can be seen in Figure 3. The optimal switching time with infinite reservoirs (but finite $C$ for working fluid) for the parameters in Figure 3 is approximately 0.792, whereas the corresponding Curzon-Ahlborn expression, (37) (infinite reservoirs and infinite $C$ working fluid), will yield about 0.828. In short, from numerical results we observe that optimal switching time varies significantly from the above limiting values if the reservoirs and working fluid are finite.

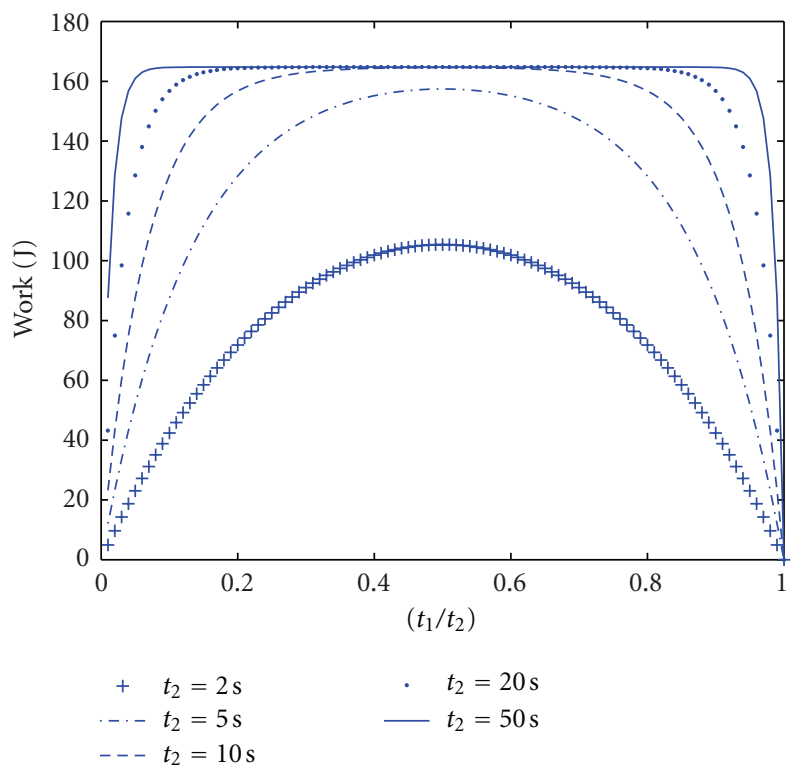

Figure 2: Optimal work as function of $t_{1} / t_{2}$. Here, $C=2 \mathrm{~J} / \mathrm{K}$; $T_{1}(0)=700 \mathrm{~K} ; T_{2}(0)=300 \mathrm{~K} ; K_{1}=3 \mathrm{~J} / \mathrm{Ksec} ; K_{2}=3 \mathrm{~J} / \mathrm{K} \mathrm{sec} ;$ $C_{1}=300 \mathrm{~J} / \mathrm{K} ; C_{2}=500 \mathrm{~J} / \mathrm{K}$.

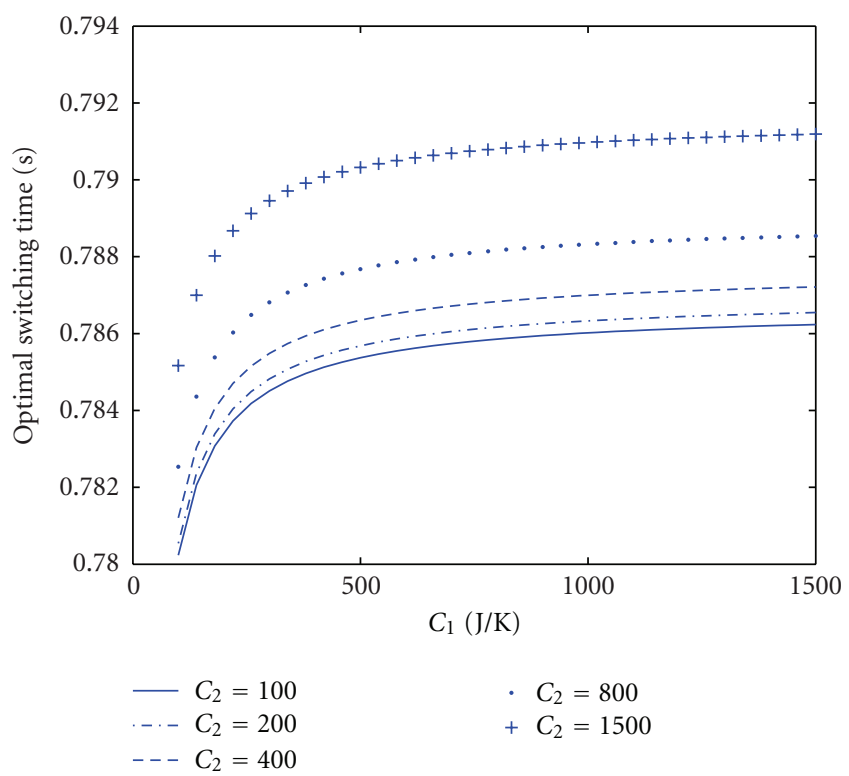

Figure 3: Optimal switching time versus the heat capacity of the hot reservoir. Here, $C=2 \mathrm{~J} / \mathrm{K} ; T_{1}(0)=700 \mathrm{~K} ; T_{2}(0)=300 \mathrm{~K} ; K_{1}=$ $6 \mathrm{~J} / \mathrm{K} \mathrm{sec} ; K_{2}=3 \mathrm{~J} / \mathrm{K} \mathrm{sec} ; t_{2}=2 \mathrm{sec}$.

4.1. Infinite Time Limit. Further, if in (33), we keep the constraints of finite reservoirs, but let the nonadiabatic branches proceed very slowly $\left(t_{1} \rightarrow \infty\right.$ and $\left.\left(t_{2}-t_{1}\right) \rightarrow \infty\right)$, we get the following expression for the maximum possible work that can be extracted from the heat engine:

$$
\widehat{W}_{\mathrm{opt}}=\frac{C\left(\sqrt{T_{1}(0)}-\sqrt{T_{2}(0)}\right)^{2}}{\left(1+\left(C / C_{1}\right)+\left(C / C_{2}\right)\right)} .
$$


The factor $1 /\left(1+\left(C / C_{1}\right)+\left(C / C_{2}\right)\right)$ accounts for finiteness of the reservoirs but with an infinite cycle time. In the limit of both infinite reservoirs as well as infinitely slow cycles, the above expression for the maximum work goes back to the one derived by Leff [11] (particularly Otto cycle and JouleBrayton cycle),

$$
\widehat{W}_{\mathrm{opt}}=C\left(\sqrt{T_{1}(0)}-\sqrt{T_{2}(0)}\right)^{2}
$$

where $C$ is the heat capacity of the working fluid.

\section{Summary}

In this paper, the maximum power point characteristics of a generalized four-step heat engine are studied. The heat cycle has two adiabatic steps and in the remaining two steps the working fluid follows a polytropic process with a constant heat capacity. It is observed that some common heat cycles such as Otto cycle, Joule-Brayton cycle, and Carnot cycle can be incorporated as special cases of this model. Curzon and Ahlborn in their seminal paper derived the expression for efficiency at maximum power for a Carnotlike engine with infinite reservoirs. Later, Gordon proved that even with finite reservoirs, Carnot-like engines show this efficiency at maximum power. Here we have analysed this result for the generalised heat cycle and shown that CA efficiency is obtained by optimizing the initial temperature of the working fluid and is independent of the switching time. However, we can further maximize this work over the switching time. Finally, the effect of finiteness of cycle time alone, the finiteness of the reservoirs alone, and the finiteness of both the cycle time and the reservoirs together on the maximum work per cycle can be respectively attributed to the following factors:

$$
\begin{gathered}
\frac{\left(1-e^{-\left(K_{1} / C\right) t_{1}}\right)\left(1-e^{-\left(K_{2} / C\right)\left(t_{2}-t_{1}\right)}\right)}{1-e^{-\left(K_{1} / C\right) t_{1}-\left(K_{2} / C\right)\left(t_{2}-t_{1}\right)}}, \\
\frac{1}{\left(1+\left(C / C_{1}\right)+\left(C / C_{2}\right)\right)}, \\
\frac{C_{1} C_{2}\left(1-e^{-\lambda t_{1}}\right)\left(1-e^{-\beta\left(t_{2}-t_{1}\right)}\right)}{C C_{1}\left(1-e^{-\lambda t_{1}}\right)+C C_{2}\left(1-e^{-\beta\left(t_{2}-t_{1}\right)}\right)+C_{1} C_{2}\left(1-e^{-\lambda t_{1}-\beta\left(t_{2}-t_{1}\right)}\right)} .
\end{gathered}
$$

It can be easily shown (see the appendix) that all these factors are less than unity, and thus, the irreversibilities due to the finiteness of time and/or the reservoirs actually reduce the maximum work that can be performed by these model engines, over the infinite-time and infinite-reservoir models.

\section{Appendix}

\section{A. Time- and Reservoir-Irreversibility Factors Are Less Than Unity}

A.1. Finite Cycle-Time Irreversibility. The factor in (45) is attributed to the finite cycle-time irreversibility. Let
$e^{-\left(K_{1} / C\right) t_{1}}=a$ and $e^{-\left(K_{2} / C\right)\left(t_{2}-t_{1}\right)}=b$. Since $K_{1}, K_{2}, C, t_{1}$, $\left(t_{2}-t_{1}\right)$ are all greater than zero, we have $0<a<1$ and $0<b<1$. It follows that $a b<b$ and $a b<a$. Adding the last two inequalities, we get $2 a b<a+b$, which may be rewritten as $(1-a-b+a b)<1-a b$. So finally, we get

$$
\frac{(1-a)(1-b)}{1-a b}<1
$$

Thus we see that the finite cycle-time irreversibility factor is less than one.

A.2. Finite-Reservoir Irreversibility. Since $C, C_{1}, C_{2}$ are all greater than zero, we have

$$
\frac{1}{\left(1+\left(C / C_{1}\right)+\left(C / C_{2}\right)\right)}<1 .
$$

A.3. Finite-Time and Finite-Reservoir Irreversibility. Using similar procedure as to time irreversibility, we can show that

$$
\frac{C_{1} C_{2}\left(1-e^{-\lambda t_{1}}\right)\left(1-e^{-\beta\left(t_{2}-t_{1}\right)}\right)}{C_{1} C_{2}\left(1-e^{-\lambda t_{1}-\beta\left(t_{2}-t_{1}\right)}\right)}<1 .
$$

Since, $C_{1}, C_{2}, C, \lambda, \beta, t_{1},\left(t_{2}-t_{1}\right)>0$, therefore, $(1-$ $\left.e^{-\lambda t_{1}}\right)>0$ and $\left(1-e^{-\beta\left(t_{2}-t_{1}\right)}\right)>0$. So, adding $\left(C C_{1}(1-\right.$ $\left.\left.e^{-\lambda t_{1}}\right)+C C_{2}\left(1-e^{-\beta\left(t_{2}-t_{1}\right)}\right)\right)$ to the denominator of left hand expression in (A.3) will reduce the expression further and retain the inequality. Thus we conclude

$$
\begin{aligned}
& \frac{C_{1} C_{2}\left(1-e^{-\lambda t_{1}}\right)\left(1-e^{-\beta\left(t_{2}-t_{1}\right)}\right)}{C C_{1}\left(1-e^{-\lambda t_{1}}\right)+C C_{2}\left(1-e^{-\beta\left(t_{2}-t_{1}\right)}\right)+C_{1} C_{2}\left(1-e^{-\lambda t_{1}-\beta\left(t_{2}-t_{1}\right)}\right)} \\
& \quad<1 .
\end{aligned}
$$

\section{Acknowledgments}

This work was initiated during the summer program at IISER Mohali. A. Khanna expresses his gratitude towards IISER Mohali, for hospitality and financial support. R. S. Johal acknowledges financial support from the Department of Science and Technology, India, under the Research Project no. SR/S2/CMP-0047/2010(G).

\section{References}

[1] F. L. Curzon and B. Ahlborn, "Efficiency of a Carnot engine at maximum power output," American Journal of Physics, vol. 43, no. 1, p. 22, 1975.

[2] D. Gutkowicz-Krusin, I. Procaccia, and J. Ross, "On the efficiency of rate processes. Power and efficiency of heat engines," The Journal of Chemical Physics, vol. 69, no. 9, pp. 3898-3906, 1978.

[3] M. H. Rubin, "Optimal configuration of a class of irreversible heat engines. I," Physical Review A, vol. 19, no. 3, pp. 12721276, 1979.

[4] M. H. Rubin, "Optimal configuration of a class of irreversible heat engines. II," Physical Review A, vol. 19, no. 3, pp. 12771289, 1979. 
[5] M. J. Ondrechen, B. Andressen, M. Mozurkewich, and R. S. Berry, "Maximum work from a finite reservoir by sequential Carnot cycles," American Journal of Physics, vol. 49, p. 681, 1981.

[6] P. Salamon, Y. B. Band, and O. Kafri, "Maximum power from a cycling working fluid," Journal of Applied Physics, vol. 53, no. 1, pp. 197-202, 1982.

[7] M. J. Ondrechen, M. H. Rubin, and Y. B. Band, "The generalized Carnot cycle: a working fluid operating in finite time between finite heat sources and sinks," The Journal of Chemical Physics, vol. 78, no. 7, pp. 4721-4727, 1983.

[8] J. M. Gordon, "Maximum power point characterstics of heat engines as a general thermodynamic problem," American Journal of Physics, vol. 57, p. 1136, 1989.

[9] L. Chen, F. Sun, and C. Wu, "Effect of heat transfer law on the performance of a generalized irreversible Carnot engine," Journal of Physics D, vol. 32, no. 2, pp. 99-105, 1999.

[10] L. Chen, S. Zhou, F. Sun, and C. Wu, "Optimal configuration and performance of heat engines with heat leak and finite heat capacity," Open Systems and Information Dynamics, vol. 9, no. 1, pp. 85-96, 2002.

[11] H. S. Leff, "Thermal Efficiency at maximum work output: new results for old heat engines," American Journal of Physics, vol. 55, p. 602, 1987.

[12] C. Van den Broeck, "Thermodynamic efficiency at maximum power," Physical Review Letters, vol. 95, Article ID 190602, 2005.

[13] Z. C. Tu, "Efficiency at maximum power of Feynman's ratchet as a heat engine," Journal of Physics A, vol. 41, Article ID 312003, 2008.

[14] M. Esposito, K. Lindenberg, and C. Van den Broeck, "Thermoelectric efficiency at maximum power in a quantum dot," Physical Review Letters, vol. 102, Article ID 130602, 2009.

[15] Y. Zhou and D. Segal, "Minimal model of a heat engine: information theory approach," Physical Review E, vol. 82, Article ID 011120, 2010.

[16] R. S. Johal, "Universal Efficiency at optimal work with Bayesian statistics," Physical Review E, vol. 82, Article ID 061113, 2010. 

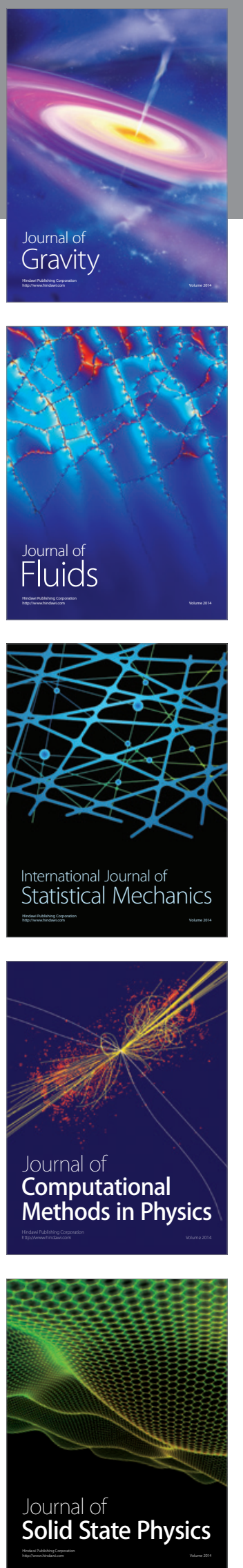

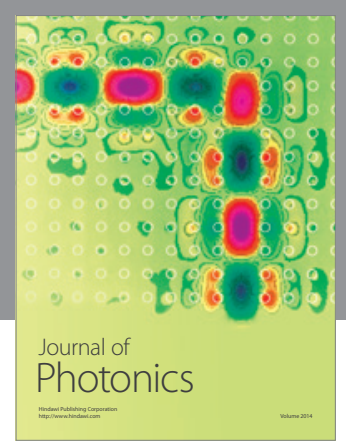

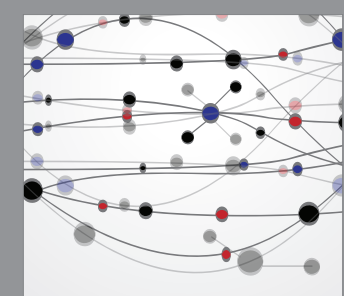

The Scientific World Journal
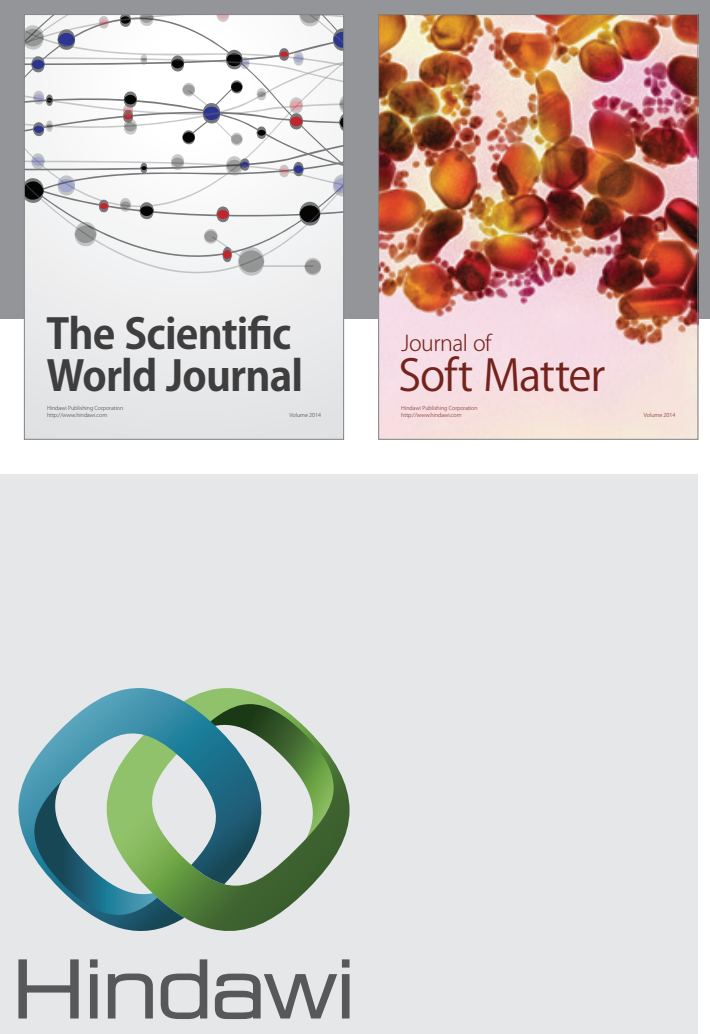

Submit your manuscripts at

http://www.hindawi.com
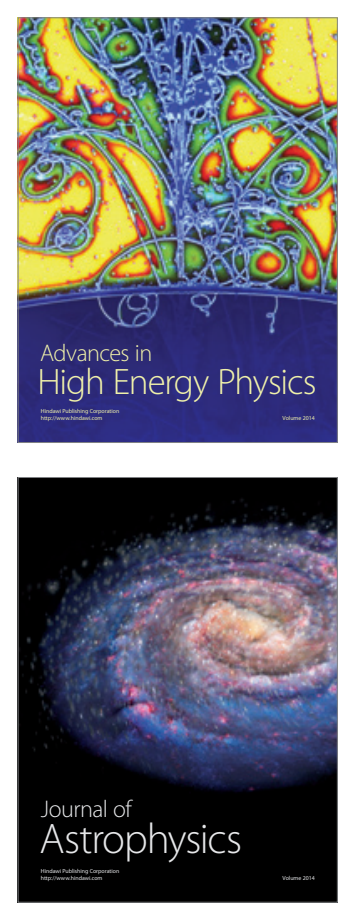
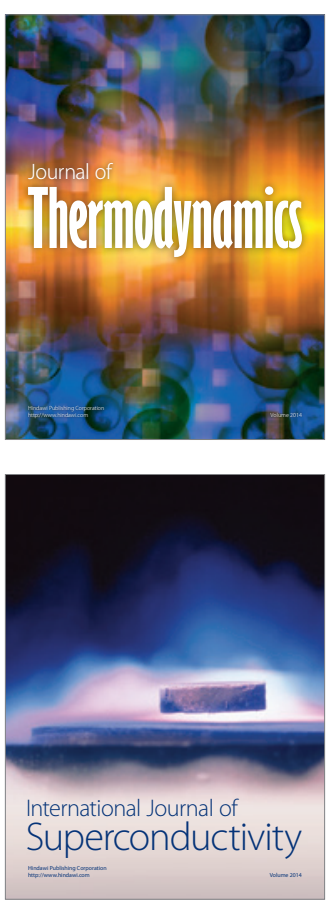
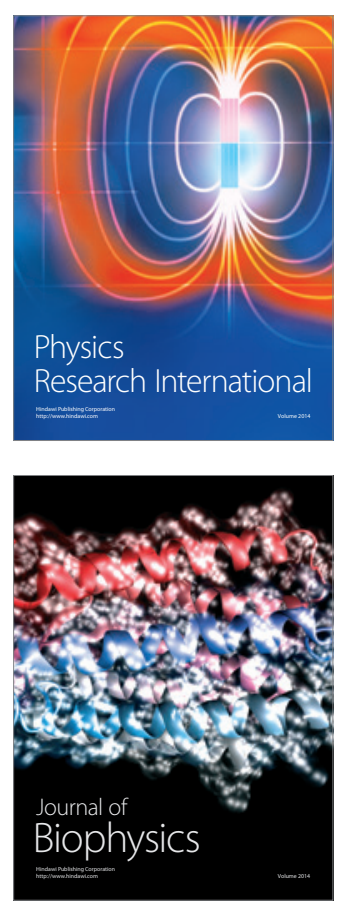
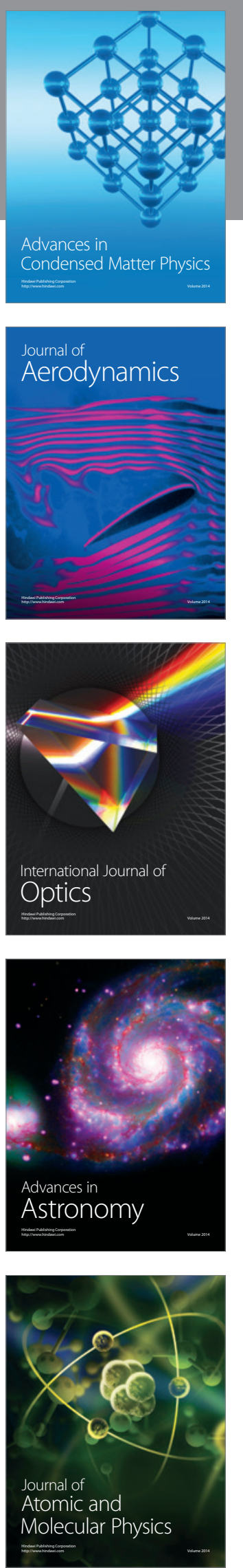